\title{
SIMULAÇÃO DO VAZAMENTO DE ÓLEO A PARTIR DE UM RECIPIENTE AFUNILADO SUBMERSO EM ÁGUA
}

\author{
T. SIMIONI ${ }^{1}$, M. B. QUADRI ${ }^{1}$, N. ULIAN ${ }^{1}$, D. R. SIMIONI ${ }^{1}$ \\ ${ }^{1}$ Universidade Federal de Santa Catarina, Departamento de Engenharia Química e \\ Engenharia de Alimentos \\ E-mail para contato: taysnarasimioni@gmail.com, mbq1959@hotmail.com
}

\begin{abstract}
RESUMO - O vazamento de óleo, muitas vezes presente nas operações offshore da indústria do petróleo, apresenta uma série de consequências negativas, tanto ambientais como econômicas. A Fluidodinâmica Computacional (Computational Fluid Dynamics - CFD) aplicada aos escoamentos multifásicos tem-se mostrado muito útil para o estudo desse tipo de problema, permitindo descrever os padrões de escoamento do óleo bem como prever os volumes de óleo vazado em caso de acidente. Com esta motivação, propõe-se a modelagem e a simulação do vazamento de óleo a partir de um recipiente submerso em água segundo a geometria de um funil com o bico virado para cima. Para isso, além das equações de Navier-Stokes e da equação da conservação da massa, emprega-se o método Level Set para acompanhamento da interface. A técnica numérica utilizada é a de elementos finitos. Os resultados obtidos envolvendo a cinética de vazamento se mostraram adequados frente aos resultados experimentais.
\end{abstract}

\section{INTRODUÇÃO}

O vazamento de óleo no oceano é um dos temas mais preocupantes no âmbito da poluição marítima, pois causa grandes danos à vida marinha, à saúde humana, e aos recursos naturais (Zhu et al., 2014). Acidentes envolvendo a captura de óleo podem ocorrer não apenas nas unidades de produção offshore (plataformas e navios de produção), como também no transporte do óleo, seja ele através de dutos ou de navios. A recuperação do ambiente afetado pode levar dezenas de anos, e os danos ao ambiente podem ser irreversíveis, afetando as esferas econômica, ecológica e social (Feller, 2012).

Uma previsão rápida e precisa do derramamento e dispersão do óleo poderia fornecer informações úteis para a criação de barreiras de contenção a fim de minimizar as consequências ambientais e os prejuízos financeiros gerados por estes acidentes (Oliveira, 2009; Zhu et al., 2014). Neste contexto, a Fluidodinâmica Computacional (CFD) tem-se apresentado como uma ferramenta muito útil, pois permite, não só a monitorização e acompanhamento da situação, mas também a previsão do comportamento do óleo derramado nas horas seguintes (Lamine e Xiong, 2013; Pierozan, 2011).

A ferramenta CFD utiliza-se de recursos computacionais e engloba um conjunto de técnicas matemáticas, numéricas e computacionais empregadas para realizar o estudo preditivo dos fenômenos de transporte (Mariano, 2008; Pierozan, 2011). A realização de simulações apresenta diversas vantagens quando comparada com a realização de testes reais, pois normalmente o desenvolvimento das simulações computacionais é muito mais barato e 
mais rapidamente reproduzido. Além disso, a simulação CFD fornece informações mais detalhadas do que as obtidas através das medições e permite ainda simular condições difíceis de serem geradas na prática (Pierozan, 2011).

São vários os softwares disponíveis no mercado que permitem a modelagem e simulação de fenômenos físicos. A escolha do software depende da avaliação de uma série de fatores: facilidade de uso, capacidade de gerar geometrias e malhas, eficiência e robustez das técnicas numéricas implementadas, etc (Feller, 2012). Dentre as opções, o software COMSOL Multiphysics merece destaque devido ao fato de apresentar diversos métodos e modelos numéricos capazes de simular escoamentos de forma bastante real.

Os modelos baseiam-se nas equações de conservação de massa, momento de impulso (Navier-Stokes) e energia (Oliveira et al., 2009). O solver deste software baseia-se no Método dos Elementos Finitos (MEF) para a discretização e resolução das simulações, o que proporciona a obtenção de resultados precisos (Feller, 2012; Pierozan, 2011).

O presente trabalho propõe a modelagem e a simulação do vazamento de óleo a partir de um recipiente submerso em água segundo a geometria de um funil com o bico virado para cima. Para tanto, utilizou-se o método Level Set para acompanhamento da inferface, através do software COMSOL Multiphysics versão 4.3 b. Além disso, pretende-se avaliar a viabilidade da modelagem frente a um estudo experimental, considerando as mesmas condições do problema simulado.

\section{MATERIAIS E MÉTODOS}

\subsection{Estudo experimental}

A obtenção de dados experimentais, para dar suporte ao desenvolvimento de um modelo computacional adequado, fez-se através da montagem de um aparato para a visualização dos fenômenos envolvidos no vazamento de óleo a partir de um recipiente submerso em água destilada. Trata-se de um funil de vidro, com o bico voltado para cima, inicialmente preenchido com óleo Spindura na temperatura ambiente $\left(\sim 20{ }^{\circ} \mathrm{C}\right)$.

A haste do funil utilizado (parte mais fina) tem diâmetro interno de 5,8 $\mathrm{mm}$ e altura de 70,0 mm. Já o bocal do funil (parte cônica) apresenta diâmetro interno de 70,7 mm e altura de $60,0 \mathrm{~mm}$. O recipiente utilizado nos experimentos apresenta diâmetro interno de $17 \mathrm{~cm}$ e utilizou-se uma altura de água destilada de $21,5 \mathrm{~cm}$.

A fim de melhorar a visualização da interface, foi adicionado ao óleo Spindura, tinta a óleo da cor azul (Acrilex). A Figura 1 apresenta uma imagem do aparato experimental montado. 


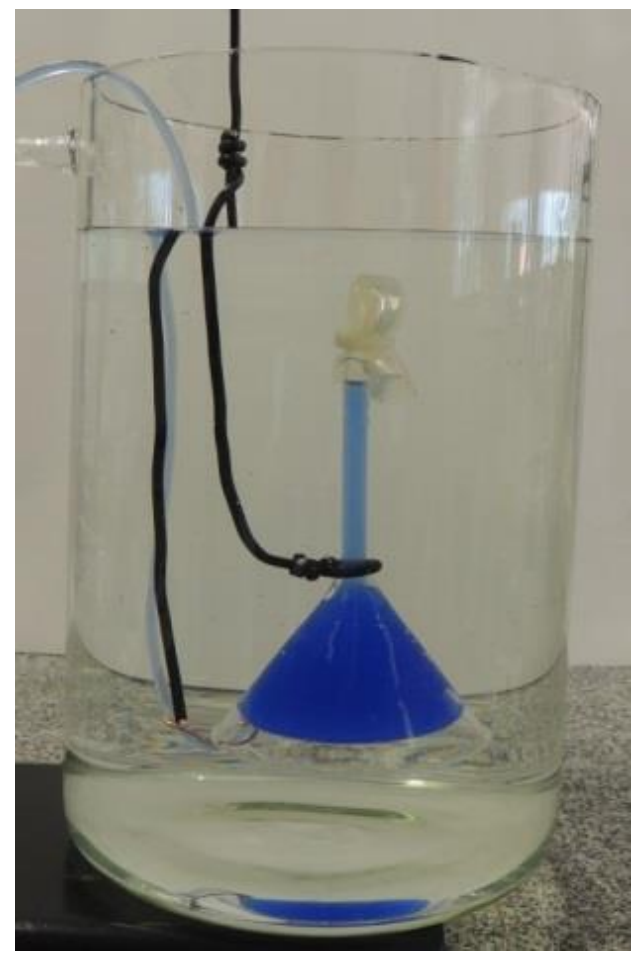

Figura 1 - Aparato experimental: funil invertido submerso em água e retendo óleo

\subsection{Estudo computacional}

As simulações apresentadas neste trabalho foram desenvolvidas através do software COMSOL Multiphysics versão 4.3b. Em todas as simulações, assumiu-se que o escoamento é laminar e isotérmico $\left(20^{\circ} \mathrm{C}\right)$. A discretização das equações diferenciais que regem o fenômeno é feita através da técnica dos elementos finitos.

A interface do escoamento bifásico água-óleo foi modelada através do método Level Set, no qual a interface é representada por um isocontorno de uma função global definida, a função Level Set $\varphi$. A $\varphi$ varia de 0 em um domínio até 1 no outro, onde através da interface, existe uma transição suave entre esses dois valores (COMSOL Multiphysics v. 4.3 b, 2014; Olsson e Kreiss, 2005; Feller, 2012).

Equações governantes: Para o escoamento laminar, envolvendo duas fases, com a interface governada pelo modelo Level Set, o transporte de massa e momento é regido pelas equações incompressíveis de Navier-Stokes apresentadas pelas Equações 1 e 2, respectivamente.

$$
\begin{aligned}
& \rho \frac{\partial u}{\partial t}+\rho(u \cdot \nabla) u=\nabla \cdot\left[-p I+\mu\left(\nabla u+\nabla u^{T}\right)\right]+F_{g}+F_{s t}+F_{\text {ext }}+F \\
& \nabla \cdot u=0
\end{aligned}
$$

Onde u é a velocidade $(\mathrm{m} / \mathrm{s}), \rho$ é a densidade $\left(\mathrm{kg} / \mathrm{m}^{3}\right), \mathrm{p}$ é a pressão $(\mathrm{Pa})$, I é a matriz identidade, $\mu$ é a viscosidade dinâmica (Pa.s), T é a temperatura $(\mathrm{K}), \mathrm{F}_{\mathrm{s}}$ é a força da gravidade 
$\left(\mathrm{N} / \mathrm{m}^{3}\right), \mathrm{F}_{\mathrm{st}}$ é a força de tensão superficial na interface dos dois fluidos $\left(\mathrm{N} / \mathrm{m}^{3}\right), \mathrm{F}_{\text {ext }}$ é a força proveniente da energia livre externa $\left(\mathrm{N} / \mathrm{m}^{3}\right)$ e F é a campo vetorial de força $\left(\mathrm{N} / \mathrm{m}^{3}\right)$.

O modelo Level Set acrescenta ainda a Equação 3, em sua forma não conservativa, que rege a $\varphi$ e oferece a possibilidade de determinação dos parâmetros associados à esta função.

$$
\frac{\partial \varphi}{d t}+u \cdot \nabla \varphi=\gamma \nabla \cdot\left(\varepsilon \nabla \varphi-\varphi(1-\varphi) \frac{\nabla \varphi}{|\nabla \varphi|}\right)
$$

O parâmetro $\varepsilon$, determina a espessura da região onde a $\varphi$ varia de zero a um. $O$ parâmetro $\gamma$ determina a quantidade de reinicializações ou estabilizações da $\varphi$. Esse parâmetro precisa ser ajustado para cada problema específico, pois está relacionado com a estabilidade da interface (COMSOL Multiphysics v. 4.3 b, 2014; Olsson e Kreiss, 2005; Feller, 2012).

Definição do modelo: Para a implementação das equações apresentadas no item anterior, o primeiro passo é a criação de uma geometria adequada para o problema. A geometria foi construída com base no modelo experimental, a fim de obterem-se resultados de simulação comparáveis aos dados experimentais, conforme Figura 2.
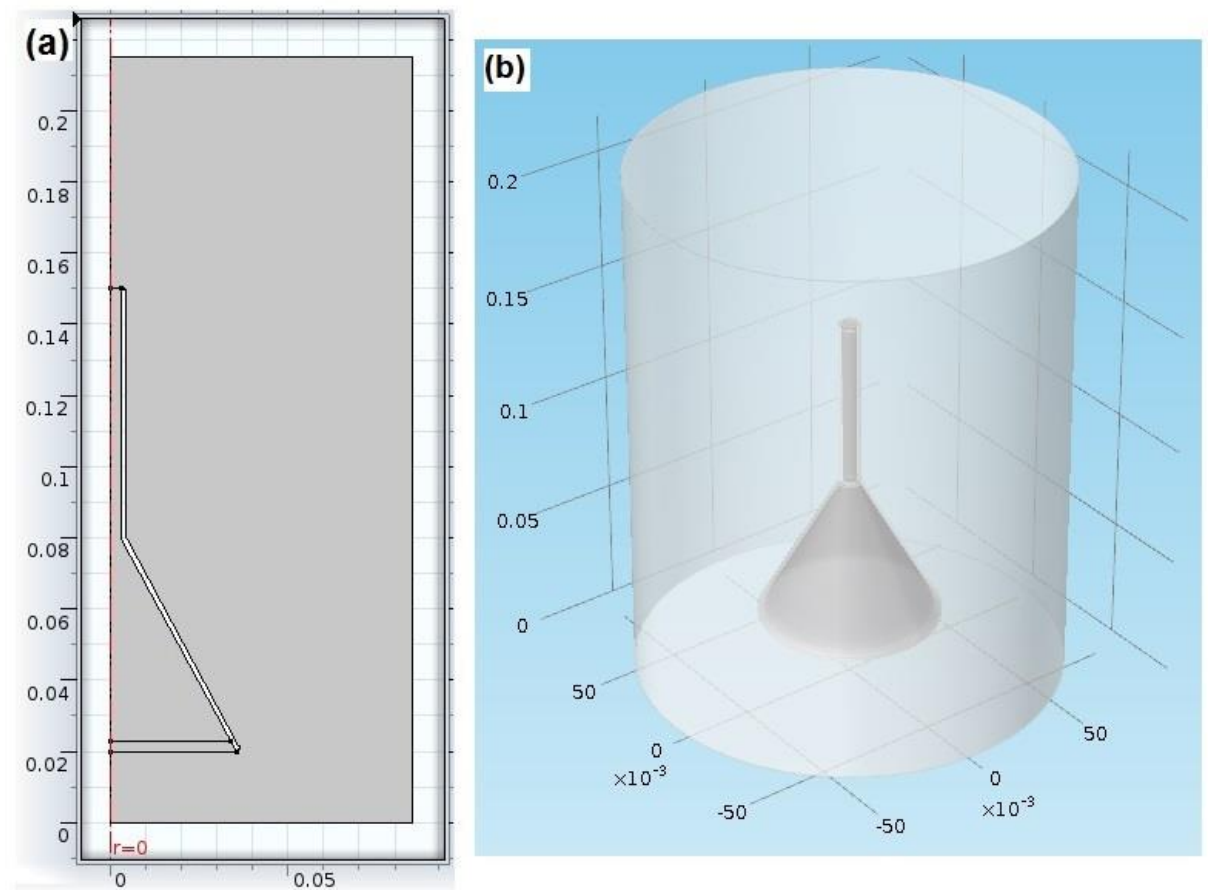

Figura 2 - Geometria: (a) 2D axissimétrico e (b) 3D

A geometria é composta por dois domínios. O domínio 1 corresponde ao Béquer que contém água destilada. $\mathrm{O}$ domínio 2 corresponde ao funil e está, inicialmente, preenchido por óleo Spindura.

Propriedades dos materiais: Utilizaram-se as mesmas propriedades dos fluidos empregados nos ensaios experimentais, previamente determinadas a $\sim 20^{\circ} \mathrm{C}$. Para o óleo Spindura, considerou-se viscosidade de $0,0175 \mathrm{~Pa} \cdot \mathrm{s}$ e densidade de $830 \mathrm{~kg} / \mathrm{m}^{3}$. Já para a água 
destilada, foi considerada viscosidade de $0,0010 \mathrm{~Pa} \cdot \mathrm{s}$ e densidade de $998 \mathrm{~kg} / \mathrm{m}^{3}$. Para a tensão interfacial água destilada/óleo Spindura adotou-se 0,03291 N/m.

Parâmetros do Level Set: Para o problema em questão, adotou-se o valor de $1 \mathrm{~m} / \mathrm{s}$ para $\gamma$ e tpf.hmax/2 m para $\varepsilon$ (tpf.hmax corresponde à maior dimensão do maior elemento da malha); ambos correspondem ao valor de default sugerido pelo software.

Condições de contorno: Uma condição de parede molhada (ângulo de contato da interface água/óleo com a parede de vidro igual a $90^{\circ}$ ) na face interna do funil foi adotada. Nas demais paredes, utilizou-se a condição de não deslizamento. Considerou-se que, inicialmente, o sistema está em repouso, adotando-se um perfil de pressão vertical ao longo dos domínios, conforme apresentado na Tabela 1, de modo a considerar o efeito da gravidade.

Tabela 1 - Perfil de pressão nos domínios do problema

\begin{tabular}{lll}
\hline & Perfil de pressão & Unidade \\
\hline Domínio 1 & tpf.rho1*g_const* $(0.4-\mathrm{z})$ & $\mathrm{Pa}$ \\
Domínio 2 & tpf.rho1 $*$ g_const $*(0.4-\mathrm{z})+$ tpf.rho2 $*$ g_const $*(0.2-\mathrm{z})$ & $\mathrm{Pa}$ \\
\hline
\end{tabular}

Definição da malha: A malha construída para o domínio em estudo está apresentada na Figura 3. Foi definida uma malha normal, composta por 9167 elementos.

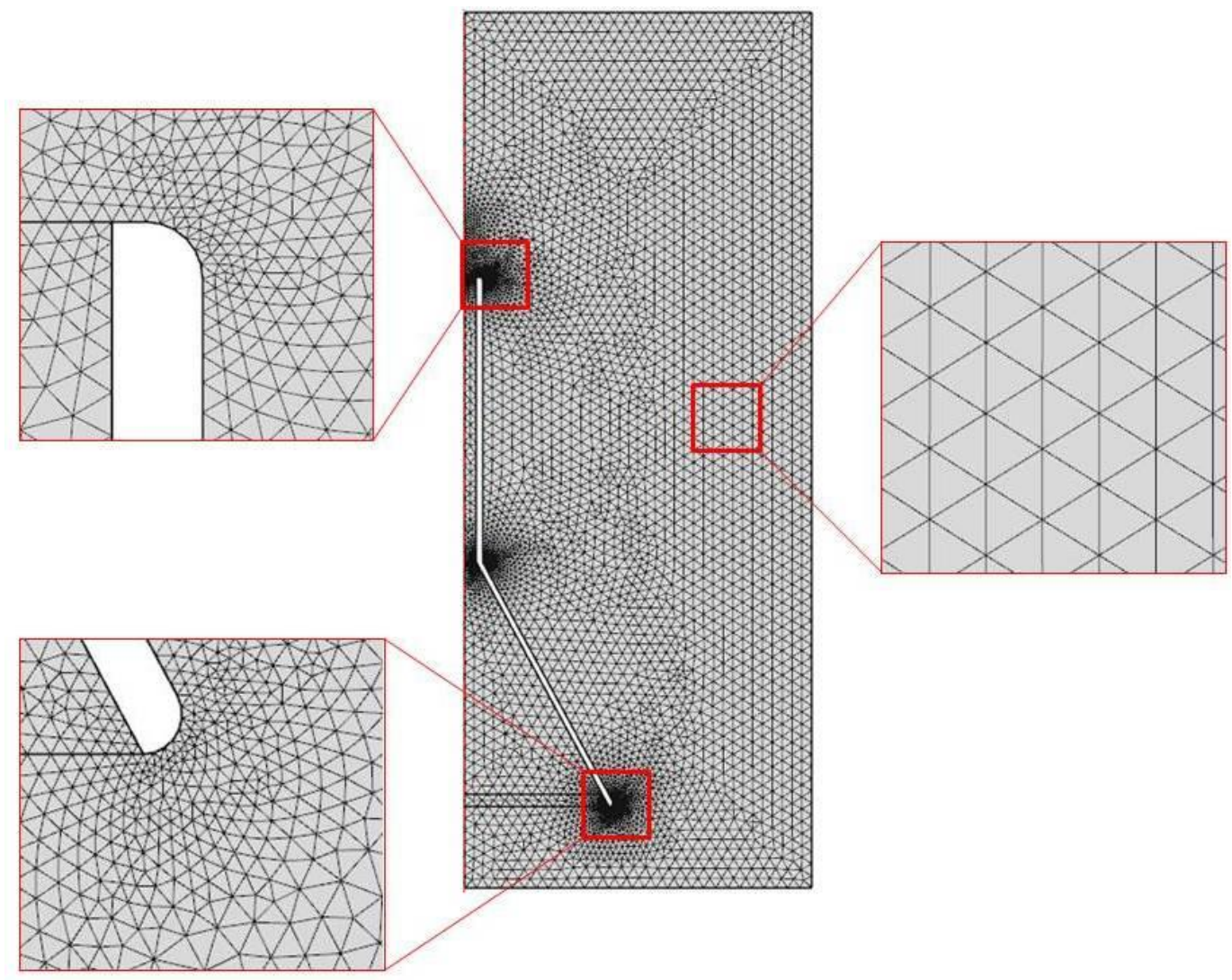

Figura 3 - Malha gerada para a solução do problema 


\section{RESULTADOS E DISCUSSÃO}

A Figura 4 apresenta a evolução do escoamento com o tempo observado tanto no ensaio experimental, quanto na simulação.

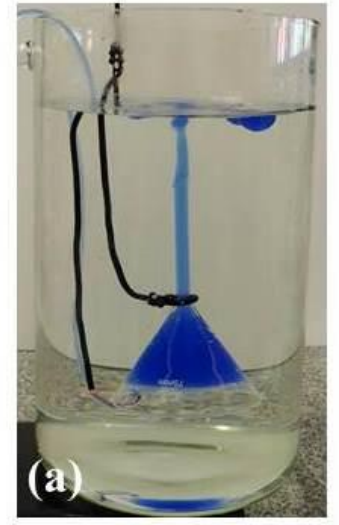

$t=4 s$

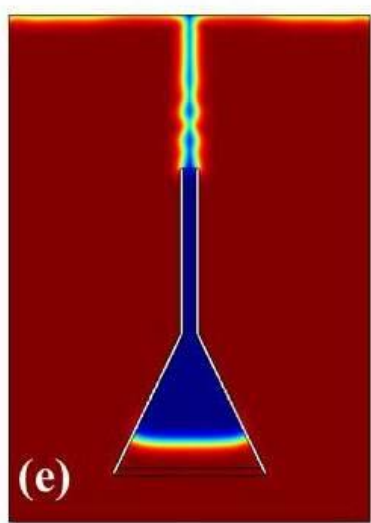

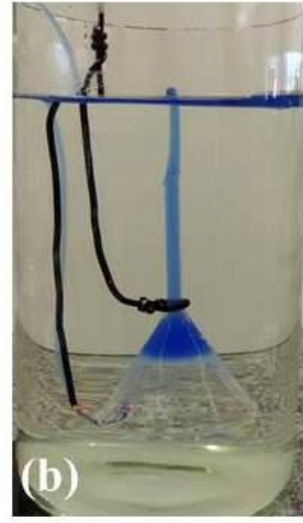

$\mathbf{t}=\mathbf{9}$ s

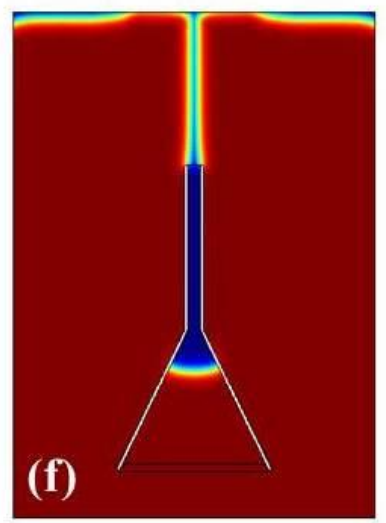

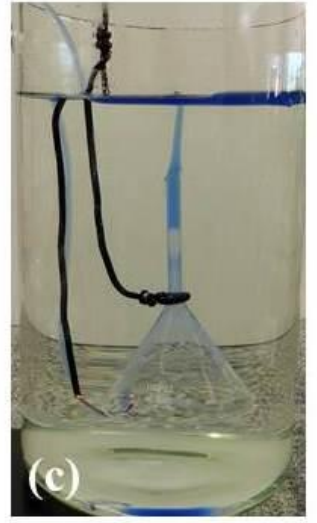

$\mathbf{t}=\mathbf{1 0} \mathrm{s}$

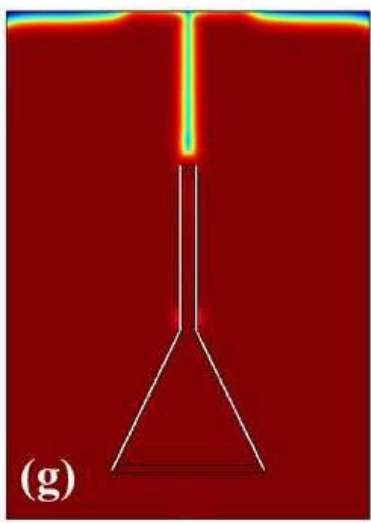

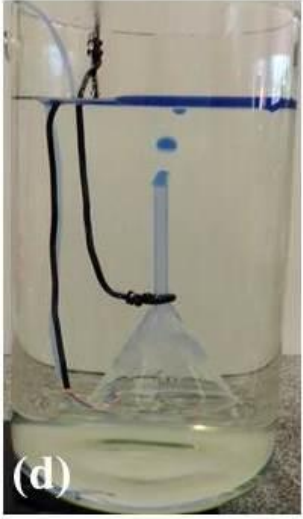

$\mathbf{t}=12 \mathrm{~s}$

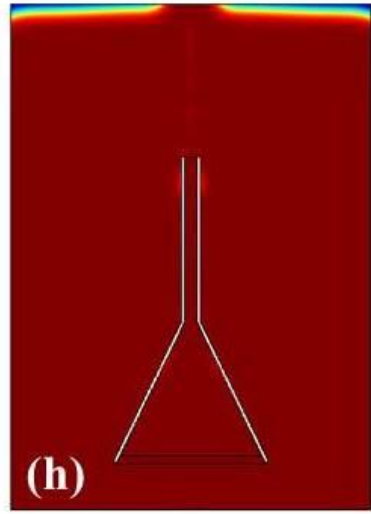

Figura 4 - Evolução do escoamento para diferentes tempos: experimental (a), (b), (c), (d) e simulado (e), (f), (g), (h)

O escoamento do óleo produzido na simulação foi muito semelhante ao observado experimentalmente. Nos primeiros 9 segundos, não são observadas divergências qualitativas significativas entre os dois estudos. Passado esse tempo, o experimental apresenta algumas gotas aderidas à parede do funil, que se desprendem vagarosamente, o que pode estar sendo produzido pela composição das forças interfaciais água/óleo e forças de adesão desses fluidos com a parede de vidro (Oliveira et al., 2009).

De acordo com Zhu et al. (2014), o escoamento de óleo é diretamente influenciado pela ação conjunta da gravidade, força inercial, flutuabilidade e tensão de cisalhamento. Feller (2012) afirma que, na ausência de forças externas, a diferença de densidades entre a água e o óleo é a principal promotora do deslocamento das fases. Isto está de acordo com o pode ser observado na Figura 4, onde o óleo, menos denso que a água, ascende e ocupa a posição superior à medida que deixa o recipiente submerso. 
A cinética de vazamento está apresentada na Figura 5 através de um gráfico comparativo entre as vazões de óleo observadas no estudo experimental e na simulação.

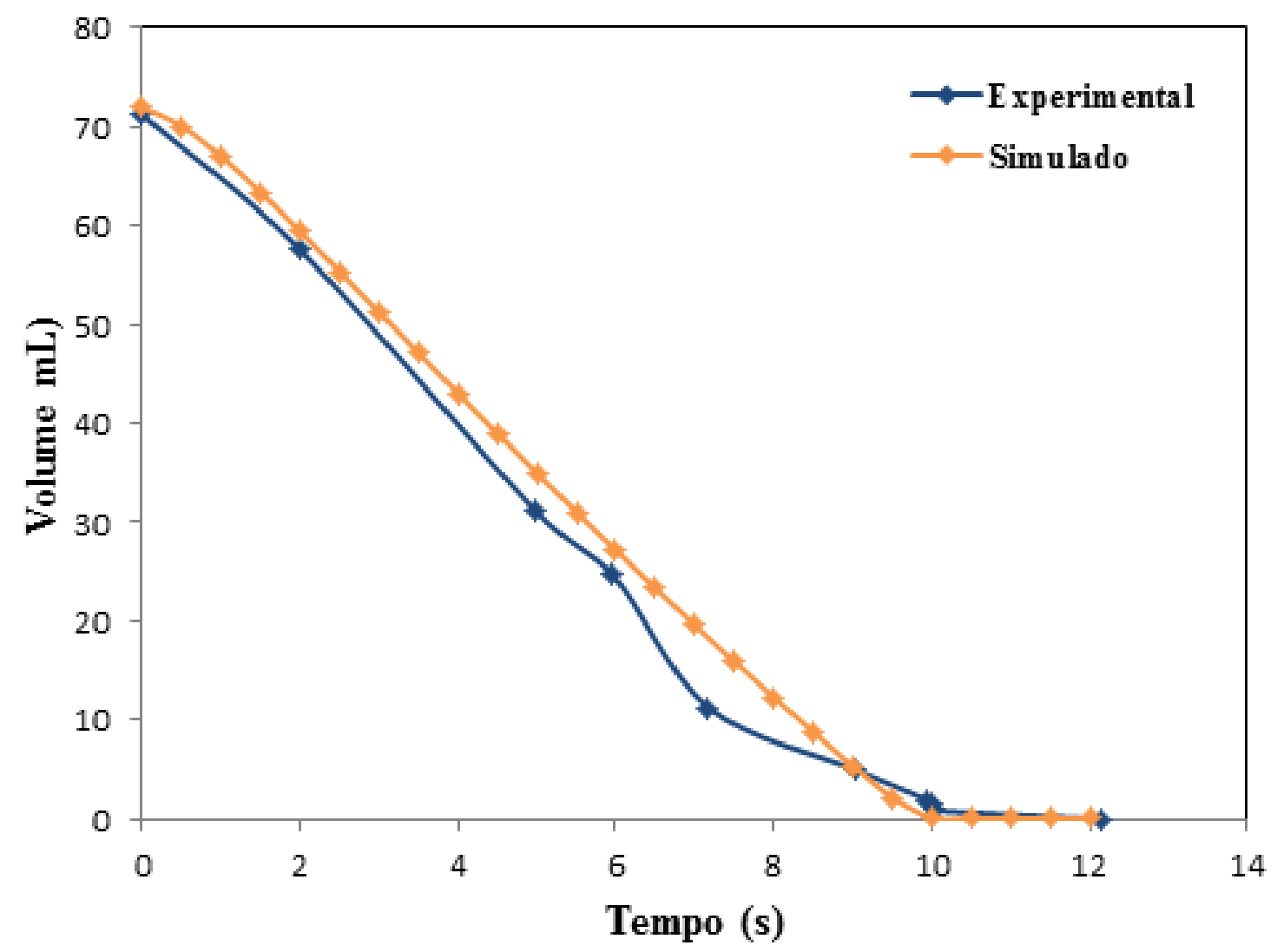

Figura 5 - Gráfico comparativo das vazões observadas

Os dados de vazão coletados no estudo experimental e na simulação são bastante próximos, validando o método empregado. Já no início do vazamento, verifica-se que a vazão de óleo tende a se tornar constante, seguindo assim até que quase todo o óleo tenha se esgotado. Para o caso experimental, os últimos pontos evidenciam uma queda da vazão, possivelmente devido ao pouco óleo restante no final do vazamento e aos efeitos viscosos na seção cilíndrica do funil, não perfeitamente representados pelo modelo. A Tabela 2 apresenta a vazão média de óleo observada para os dois casos (experimental e simulado), bem como o erro experimental.

Tabela 2 - Vazão média de óleo (experimental e simulado) e desvio da simulação

\begin{tabular}{ccc}
\hline \multicolumn{3}{c}{ Vazão média (mL/s) } \\
\hline Experimental & Simulado & Desvio (\%) \\
5,8519 & 5,9879 & $+2,32$ \\
\hline
\end{tabular}

A proximidade entre os valores de vazão média experimental e simulado, com baixo desvio percentual, atestam o bom desempenho do modelo. A pequena discrepância entre os valores pode ser atribuída a perturbações externas ao sistema introduzidas durante o manuseio do material, e também, como já assinalado, devido à forma simplificada como os efeitos de viscosidade e de tensão interfacial são considerados pelo modelo. 


\section{CONCLUSÃO}

O modelo fundamentado nas equações de Navier-Stokes e no método Level Set para acompanhamento da interface mostrou-se capaz de descrever de forma coerente $\mathrm{o}$ comportamento físico do escoamento água/óleo, observado no sistema experimental. Desde o início do escoamento, o óleo apresentou a tendência de ascender e formar uma camada sobrenadante na superfície. Este é o comportamento físico esperado, devido ao óleo ser menos denso que a água e ser esta diferença de densidade a principal promotora do deslocamento das fases. O estudo computacional realizado permitiu ainda simular de modo satisfatório a cinética de vazamento do óleo. Os dados de vazão coletados no estudo experimental e na simulação foram bastante próximos, o que, aliado ao baixo desvio percentual observado entre os valores encontrados nos dois estudos, valida o método empregado.

Conclui-se que o modelo proposto tem potencial para auxiliar no desenvolvimento de ferramentas preditivas para o projeto e controle de processos envolvendo o escoamento água/óleo. Prevê-se que se aplicado em escala real, esta abordagem proporcionaria informações importantes com relação ao escoamento imiscível do óleo, auxiliando na contenção e na minimização dos prejuízos, no caso de vazamento em ambiente subaquático.

\section{REFERÊNCIAS}

COMSOL Multiphysics 4.3b. COMSOL Documentation, Março 2014.

FELLER, R. Estudo do vazamento e captura de óleo em ambiente subaquático, UFSC/Universidade Federal de Santa Catarina, Florianópolis - SC (Dissertação de mestrado), 2012.

LAMINE, S; XIONG, D. Guinean environmental impact potential risks assessment of oil spills simulation. Ocean Eng., v. 66, p. 44-57, 2013.

MARIANO, G. Estudo do escoamento imiscível água/óleo mediante experimentação em Célula de Hele-Shaw e Simulação CFD, UFSC/Universidade Federal de Santa Catarina, Florianópolis - SC (Dissertação de mestrado), 2008.

OLIVEIRA, B.; MARIANO, G.; QUADRI, M. Um estudo CFD de vazamento de óleo a partir de dutos submersos. VII Congresso Brasileiro de Engenharia Química em Iniciação Científica, 2009.

Olsson e Kreiss, 2005

PIEROZAN, M. Validação de modelo para predição do coeficiente de descarga de um tanque esférico por dinâmica de fluidos computacional, UFRGS/Universidade Federal do Rio Grande do Sul, Porto Alegre - RS (Trabalho e conclusão de curso), 2011.

ZHU, H.; LIN, P.; PAN, Q. A CFD (computational fluid dynamic) simulation for oil leakage from damaged submarine pipeline. Energy, v. 64, p. 887-899, 2014. 